\title{
Recommender Systems for Personalized Gamification
}

\author{
Gustavo F. Tondello \\ University of Waterloo \\ HCI Games Group, Cheriton School \\ of Computer Science \\ Waterloo, ON, Canada N2L 3G1 \\ gustavo@tondello.com
}

\author{
Rita Orji \\ University of Waterloo \\ HCI Games Group, Cheriton School \\ of Computer Science \\ Waterloo, ON, Canada N2L 3G1 \\ rita.orji@uwaterloo.ca
}

\author{
Lennart E. Nacke \\ University of Waterloo \\ HCI Games Group, Drama and \\ Speech Communication, Cheriton \\ School of Computer Science \\ Waterloo, ON, Canada N2L 3G1 \\ lennart.nacke@acm.org
}

\begin{abstract}
Gamification has been used in a variety of application domains to promote behaviour change. Nevertheless, the mechanisms behind it are still not fully understood. Recent empirical results have shown that personalized approaches can potentially achieve better results than generic approaches. However, we still lack a general framework for building personalized gameful applications. To address this gap, we present a novel general framework for personalized gameful applications using recommender systems (i.e., software tools and technologies to recommend suggestions to users that they might enjoy). This framework contributes to understanding and building effective persuasive and gameful applications by describing the different building blocks of a recommender system (users, items, and transactions) in a personalized gamification context.
\end{abstract}

\section{CCS CONCEPTS}

-Information systems $\rightarrow$ Personalization; Recommender systems; •Human-centered computing $\rightarrow$ User models;

\section{KEYWORDS}

Gamification, Personalization, Recommender Systems

\section{ACM Reference format:}

Gustavo F. Tondello, Rita Orji, and Lennart E. Nacke. 2017. Recommender Systems for Personalized Gamification. In Proceedings of UMAP'17 Adjunct, fuly 09-12, 2017, Bratislava, Slovakia, , 6 pages.

DOI: $10.1145 / 3099023.3099114$

\section{INTRODUCTION}

Gamification is the use of game design elements in non-game contexts [19]. It can be used to create applications aimed at promoting behaviour change in a broad range of domains, such as health, wellness, education, training, online communities, customer loyalty, marketing, and staff management [43, 58, 63]. When used for behaviour change or promotion, gameful applications usually incorporate elements of persuasive technologies $[21,51]$ in addition to the game design elements, to increase the motivational power

Permission to make digital or hard copies of all or part of this work for personal or classroom use is granted without fee provided that copies are not made or distributed for profit or commercial advantage and that copies bear this notice and the full citation on the first page. Copyrights for components of this work owned by others than the author(s) must be honored. Abstracting with credit is permitted. To copy otherwise, or republish, to post on servers or to redistribute to lists, requires prior specific permission and/or a fee. Request permissions from permissions@acm.org.

UMAP'17 Adjunct, fuly 09-12, 2017, Bratislava, Slovakia

(c) Owners/Authors, 2017. This is the author's version of the work. It is posted here for your personal use. Not for redistribution. The definitive Version of Record was published in UMAP '17 - Adjunct Publication of the 25th Conference on User Modeling, Adaptation and Personalization.

DOI: $10.1145 / 3099023.3099114$ of the persuasive intervention. Nevertheless, the mechanisms behind gameful design and gamification are still not fully understood. Empirical studies have sometimes reported only partially positive or even negative results $[27,30,51,63]$. A topic that has recently gained attention is understanding how to personalize these gameful persuasive applications. Results have shown the higher value of personalized over generic approaches in user interface (UI) design $[3,44]$, persuasive technologies [31, 33], and games [5, 14, 50, 53]. However, the study of personalized gamification is still in its infancy and publications so far have been mostly theoretical, for example, focusing on identifying different personality traits or preferences for personalization $[29,52,64]$.

On the other hand, there is a more established research literature on recommender systems (RS). RS are software tools and techniques that provide suggestions for particular items to a user [59]. A RS can help the user find items that would probably match their preferences among the increasing amount of available information and products. Additionally, a RS relies on people making choices based on what other people recommend [59]. Recommender systems have been often used to help users select products in e-commerce sites, movies, or music, just to name a few common applications.

Gameful, persuasive applications support the feeling of autonomy. They do this by offering different activities and letting users select choices $[16,60,61]$. In this regard, they share similarities with recommender systems applications. Yet, information overload may occur when options increase. This makes informed choices more difficult. In a case like this, a recommender system could help users easily choose activities that would likely fit their preferences. However, to the best of our knowledge, the current literature has not yet addressed this topic.

This paper presents the first theory on how to use recommender systems in gamification to increase their efficacy. To this end, we describe a strategy by which gameful systems can automatically recommend personalized activities for each user with the goal of increasing user motivation and engagement. We combined existing knowledge from both research fields-recommender systems and personalized gamification-to create a general framework for using recommender systems for personalized gamification. We propose a new research direction on personalized gamification using recommender systems for human-computer interaction (HCI). The framework we propose in the present work can serve as a foundation for future research. The goal of this research would be building and evaluating these systems. With our framework, researchers can describe, design, and assemble recommender system components for gamification. 


\section{RELATED WORK}

\subsection{Recommender Systems}

Recommender systems (RS) are software tools and techniques that can recommend items users might have a need for or could use [59]. They work to gather various kinds of data to build their recommendations. As a general classification, data used by RSs can refer to three kinds of entities: (1) items that are recommended to users, (2) users that will receive the recommendations, and (3) transactions (i.e., recorded interactions between items and users)[59]. There are many different ways of modelling users, which will depend on the application domain and the recommendation technique. Most personalization systems are based on some sort of user profile; this is an instance of data representing a single user [23].

More recently, RSs have also become context-aware. They consider information about a user's situation in a specific time or location that can affect the recommended items (CARS; [1]).

The task domain, the knowledge used, and the recommendation algorithm all influence the recommender system type. In detail, the algorithms vary in how they compute the rating function and how they build the recommendation and present it to the user [59]. The common recommendation techniques include:

- content-based recommendation [36, 56,59], which try to recommend items similar to those liked by the user in the past;

- collaborative (or social) filtering [17, 35, 59, 62], which recommend items that are also liked by similar users;

- hybrid recommendation $[11,59]$, which are based on the combination of the above mentioned techniques;

- context-aware recommendation [1], which consider the context in which the recommendation occurs; and

- machine-learning techniques [9], which can be used to learn models to predict ratings and are generally employed to improve the precedent techniques.

Each recommendation technique has strengths and weaknesses that influence how we select the right option for each situation. For example, content-based recommenders are better at recommending items that have enough known properties. In contrast, collaborative filtering can only recommend items that many users have rated. Hybrid methods combine the strengths of each technique to balance out the shortcomings of the others.

\subsection{Personalized Gamification}

On the other hand, personalization has been a study topic in HCI for some time (e.g., focusing on personalizing web sites or e-commerce applications) [10]. However, investigating personalization in gamification and persuasive technologies is a new field. The first empirical studies have reported that personalization is more effective than standardization to create behaviour change $[12,46]$.

Gamification adds some game elements (or motivational affordances) to a non-game application. The goal of this is to engage and motivate the user $[18,40,41]$. For example, some of the most used elements are: points, badges, leaderboards, levels, avatars, narrative, quests, challenges, and rewards. Thus, several research efforts on personalized gamification have been focused on understanding different user preferences regarding each game element or affordance.
For example, Ferro et al. [20] studied personality models and player types. The goals were to find the similarities between them and to relate them to different game design elements. Their work grouped personality traits, player types, and game elements in five categories: (1) Dominant, (2) Objectivist, (3) Humanist, (4) Inquisitive, and (5) Creative. Jia et al. [29] studied the relation between the five-factor model (FFM) personality traits $[15,25]$ and individual gamification elements and found several significant correlations. Orji et al. [52] studied the relation between the FFM personality traits and several persuasive strategies used in gamification. They noted that personalization is the most effective strategy, which almost all the personalities perceived as positive.

Marczewski [37] built on the literature about player types [8, 28, $42,67]$ and self-determination theory in games $[16,60,61]$ to create the User Types Hexad model, which classifies user preferences in six distinct user types:

- Philanthropists are motivated by purpose;

- Socialisers are motivated by relatedness;

- Free Spirits are motivated by autonomy;

- Achievers are motivated by competence;

- Players are motivated by rewards; and

- Disruptors are motivated by the triggering of change.

Tondello et al. [64] tested the correlation of each Hexad user type with several used game elements. Results demonstrated that the Hexad model could help to personalize gameful applications. The authors suggest that designers would be able to screen their target audience with the Hexad user types to choose the adequate game elements for each user.

Barata et al. [6, 7] and Von Roy and Zaman [66] studied data regarding student performance and preferences in gamified university level courses and observed evident differences.

Furthermore, adopting some sort of virtual coach or personal assistant can personalize gameful applications. This coach or assistant would be able to learn the user's preferences over time. It could then recommend interactions that the user would more likely enjoy. Application domains, such as smart communities [57] and health [65], have suggested using a coach like this. This approach would benefit from the use of recommender systems to empower the virtual assistant with useful recommendations for each user.

Gamification draws from research in persuasive technologies to further encourage adoption of behaviours. Theoretical and empirical studies have suggested different factors for persuasive technology personalization [46, 51], such as:

- Personality types $[2,4,26]$,

- Age [49],

- Gender [48, 49, 54],

- Player types [50,53],

- Culture or nationality [34, 45, 47], and

- Individual susceptibility to persuasive attempts [31, 32, 50].

To the best of our knowledge, this paper presents the first discussion of how to use recommender systems in personalized gamification. A few works discuss similar approaches; even so, there are significant differences in relation to our approach. Meder and Jain [39] studied the recommendation of game elements that would maximize each user's contribution in gameful systems using matrix factorization (a collaborative filtering technique). While their work 
overlaps with ours, it is limited to studying this single approach, whereas our work is more general. Geiger and Schader [24] reviewed the state of the art on personalized task recommendation in crowdsourcing information systems. Some crowdsourcing systems use gamification to motivate their users. Yet, crowdsourcing is one of the possible application domains for gamification, and not every crowdsourcing systems uses gamification.

In addition, there is interest from the gamification industry in building personalized solutions. For example, Gadiyar [22] argues for the need of evolving gamification practice toward personalized experiences and Paharia [55] suggests using gamification and big data analytics to build loyalty programs. Nevertheless, neither propose an actionable method to implement personalized gamification.

\section{RECOMMENDER SYSTEMS FOR PERSONALIZED GAMIFICATION}

To advance future research for building recommender systems for personalized gamification, we propose a general framework that researchers can use to design these systems. We centre this framework on the core elements of a recommender system:

Inputs: the items to be rated and recommended by the system, the user profiles, the transactions between items and users that will be logged and analyzed, and the different types of context in which the recommendations can occur.

Output: the ratings that the RS will predict.

Process: the recommendation methods of the system.

Figure 1 shows how each of the elements above contribute to the recommendation process. Our framework shows the available design options for each element. The engineers can then choose them depending on the application (e.g., by selecting which recommendation algorithm to use).

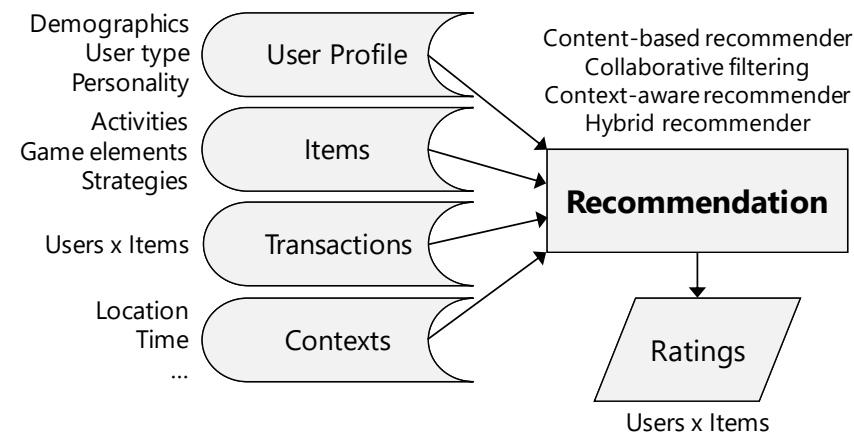

Figure 1: Inputs and outputs of the recommendation.

\subsection{Items}

The items are the user activities in the gameful application and how it communicates with and motivates the user. There might be different types of items that could be personalized in a gameful application. However, as Mattheiss et al. [38] noted, we need to choose a set of item types that will achieve a balance between personalization costs and returns. Based on the literature review documented in the previous section, we have identified three main item types to personalize, which would represent the most valuable returns: (1) the user activities, (2) the game elements that engage and motivate the user, and (3) the persuasive strategies.

3.1.1 Activities. The activities of a user in a gameful application depend on the domain. For example, in an e-commerce application, they would be browsing, visiting, and buying products; in a fitness application, they would be a variety of types of physical exercise; in a health application, they could be dieting or exercising, among others; and in a learning application, they could be reading lessons, watching videos, or taking quizzes, or completing challenges. The RS would need to rely on knowledge gathered from the application domain to analyze user preferences for different activities and recommend those that the user would be more likely to enjoy. For example, RSs in e-commerce applications have already been extensively studied and this knowledge could be used to inform the gamification RS. Similarly, much literature investigates the types of healthy activities more suited to each individual, or the differences between individual learning preferences and distinct learning activities that would be more effective for each individual. The RS can take advantage of this knowledge to recommend tailored activities according to predicted user preferences instead of suggesting the same activities to all users.

3.1.2 Game elements. There is evidence that users tend to enjoy some game elements more than others and that it is possible to assess these preferences with a user type survey [64]. Thus, the RS could recommend different types of gaming activities to satisfy the user's preferences. For example, after the RS identified that the user might enjoy carrying out a particular exercise type (e.g., walking, running, or hiking), or a particular learning activity (e.g., watching a video or taking a quiz), the system might combine it with a particular type of gaming activity that the user would be likely to enjoy, such as solving a challenge, completing a quest, competing with others, collaborating with others, or exploring different ways of completing a task.

3.1.3 Persuasive strategies. The RS can also adapt the persuasive strategies used to communicate with the user [52]. For example, using Cialdini's strategies [13], if the system finds out the user is more susceptible to scarcity, it can suggest limited opportunities to complete a task; if the user seems more susceptible to authority, the system can show expert comments for each activity; and if the user is more susceptible to consensus, the system may show that a large quantity of people are already carrying out an activity.

\subsection{Users}

Several attributes might be of use to build a user profile for the RS. For example, an important piece of information is the user type [37], which could be obtained by asking the user to fill out a survey [64]. Similarly, knowing the user's personality traits, age, gender, and nationality could be useful because there is evidence that these factors may affect a user's preferences. Additionally, there might also be attributes specific to the domain. For example, in a health application, it might be useful to know the user's health history and current condition; in a learning application, it might be useful to know about the user's current expertise on the study topic or the user's preferences for different learning styles. 


\subsection{Transactions}

In a gameful application, the transactions will be the activities actually performed by a user. Each time the user performs an activity, the RS can take the opportunity to record the user's rating for that particular activity, game element, or combination of both. These ratings can be collected implicitly (i.e., by assuming that the user carrying out an activity indicates they enjoy it, thus increasing the implicit rating for that activity and game element) or explicitly, by asking the user to rate the activity after its completion. Additionally, the RS can also rate the different persuasive strategies, either implicitly (i.e., by assuming that if the user took the suggested action, the persuasive strategy used might be a good fit) or explicitly (i.e., by asking the user about the effectiveness of each used message). It is also possible to use frequency of visit or click at a particular feature or element, or the time spend on a task or using a particular element, to determine the user's preference.

\subsection{Context Types}

The different types of context that need to be considered by the RS depend on the domain. Thus, it is also possible to learn from knowledge already documented in each specific domain to inform the RS. For example, in a health or fitness application, the season, time, or location might limit the kind of activities that the user can perform. In an e-commerce application, the location or seasonal factors may also limit the availability or cost of some products.

\subsection{Ratings}

The ratings are the output of the RS and correspond to the predicted likelihood that the user will enjoy each item not previously rated for that user. The complexity of a RS for persuasive gamification is that there are three different types of items to analyze and recommend: activities, game elements, and persuasive strategies.

In the simpler approach, the RS would receive input data about transactions and ratings for each of these types of items and would attempt to predict new ratings for activities, game elements, and persuasive strategies separately. This approach would simplify the implementation; but, it would defer the decision on how to combine these different types of items to another application component before making the recommendation to the user. Thus, the complexity is only being transferred to another subsystem, that might not have the capability to assess the user's preferences when performing the combinations.

A more comprehensive approach would be to have the RS receive ratings for each different item type separately, as well as for the specific combinations of items, then try to predict the ratings for complex combinations based on the input data. This approach would increase the complexity of the RS; however, it would enable the system to provide more accurate and useful recommendations because all the combinations between activities, game elements, and persuasive strategies will be rated according to the user preferences analyzed from the input data.

\subsection{Recommendation Methods}

The choice of the recommendation method to use will depend on the application domain and the resources available.
3.6.1 Content-based recommender. A content-based recommender can take advantage of knowledge from the application domain, if available, to implement recommendation based on the user profile and information about the items. Additionally, there is theoretical and empirical information available to enable the implementation of game elements recommendation based on the user's type [64]. The advantage of this approach is that it does not suffer from the problems of cold start or lack of coverage. However, it depends on having enough information about the user's type and the game elements themselves. Thus, this approach might not work if the user is not willing to fill out the user types survey.

3.6.2 Collaborative filtering. Collaborative filtering is also a viable solution for any of the types of items. It can overcome the lack of theoretical or empirical knowledge to build a content-based recommender. It only depends on the ratings collected explicitly or implicitly from user transactions. Yet, collaborative filtering suffers from cold start and coverage problems. A recommender algorithm will be less likely to provide good recommendations for new users (or new items) without enough initial ratings.

3.6.3 Context-aware recommender. Contextual information allows us to filter the recommended items within limitations of a domain. The decision to use a context-aware recommender will depend on the application domain and our existing knowledge about contextual information. This approach could be used together with either a content-based or a collaborative recommender system.

3.6.4 Hybrid recommender. A hybrid recommender could be used to take advantage of the best characteristics of each of the above methods. For example, a content-based recommendation algorithm can be used to provide initial recommendations based on theoretical and empirical models while a transaction history is not yet available to feed a collaborative filter. After that, the collaborative filtering algorithm might be used to improve or completely replace the content-based recommendations. Contextual information can further be used to improve or filter the recommendations based on the limitations of the application domain.

\section{CONCLUSION AND FUTURE WORK}

We have presented a new framework for building recommender systems for personalized gamification. This general framework describes the possible inputs for this type of RS (items, users, transactions, and contextual information), the possible approaches to choose a recommendation algorithm, and the process's output (the predicted ratings for each gameful activity per user). Additionally, we have described three potential types of items that can be personalized: the activities carried out by the user, the gameful elements employed to motivate the user, and the persuasive strategies used to trigger the desired behaviours. Likewise, we have described the user attributes that might be used as factors for personalization, in particular the user's gamification type scores.

Still, the currently available knowledge on personalized gamification limits our current framework. The theoretical models and empirical studies in this field will evolve in the next years. This evolution will enable more precise decisions on how to design good recommender systems for personalized gamification. 
Furthermore, we decided not to choose or test any particular approach or algorithm for building a personalized gamification recommender system to keep our framework generic. Instead, we choose to present the available options to inform future research on this topic. Future work will need to put in place and test this framework in specific application areas, by deciding on specific approaches to implement each one of its components. At this point, it is probable that new issues and questions will be encountered that our general overview did not identify.

This work contributes a new research direction on personalized gamification based on recommender systems. The proposed framework can inform the design of these systems in future research, which will then be able to evaluate the effectiveness of personalized systems over generic approaches. This novel research area opens new possibilities on investigating and building personalized persuasive gameful applications based on the empirical studies on personalized gamification described in the literature review.

Continuing this research, we plan to apply the suggested framework within different gamification projects in different domains. This will give us the opportunity to further improve the framework and to investigate specific approaches for particular domains. Moreover, we will follow with empirical user studies to evaluate the effectiveness of the recommendations for real users of gameful applications. This will allow us to further validate the framework and the specific approaches for each application domain, as well as collect valuable data to further improve the framework and advance future research on this new topic.

\section{ACKNOWLEDGMENTS}

The authors would like to thank Dr. Paulo Alencar for his input on recommender systems. Gustavo Tondello would like to thank the University of Waterloo and the CNPq, Brazil for his funding. Rita Orji would like to thank the Canadian Government Banting NSERC for her funding. Lennart Nacke and his research team would like to thank NSERC (Discovery), SSHRC (IMMERSe), and Mitacs for kindly funding this research.

\section{REFERENCES}

[1] Gediminas Adomavicius and Alexander Tuzhilin. 2011. Context-Aware Recommender Systems. In Recommender Systems Handbook, Bracha Shapira, Francesco Ricci, Paul B Kantor, and Lior Rokach (Eds.). Springer, Chapter 7, 217-253.

[2] Nurcan Alkış and Tuğba Taşkaya Temizel. 2015. The impact of individual differences on influence strategies. Personality and Individual Differences 87 (2015) 147-152. DOI : http://dx.doi.org/10.1016/j.paid.2015.07.037

[3] Ofer Arazy, Oded Nov, and Nanda Kumar. 2015. Personalityzation: UI personalization, theoretical grounding in HCI and design research. AIS Transactions on Human-Computer Interaction 7, 2 (2015), 43-69. http://aisel.aisnet.org/thci/vol7/ iss $2 / 1$

[4] Sonia M Arteaga, Mo Kudeki, Adrienne Woodworth, and Sri Kurniawan. 2010 Mobile system to motivate teenagers' physical activity. In Proceedings of the $9^{\text {th }}$ International Conference on Interaction Design and Children. ACM, 1-10. DOI : http://dx.doi.org/10.1145/1810543.1810545

[5] Sander Bakkes, Chek Tien Tan, and Yusuf Pisan. 2012. Personalised Gaming. In Proceedings of The $8^{\text {th }}$ Australasian Conference on Interactive Entertainment Playing the System - IE '12. ACM, 1-10. DOI : http://dx.doi.org/10.1145/2336727.2336731

[6] Gabriel Barata, Sandra Gama, Joaquim Jorge, and Daniel Gonçalves. 2016. Studying student differentiation in gamified education: A long-term study. Computers in Human Behavior (2016).

[7] Gabriel Barata, Sandra Gama, Joaquim A.P. Jorge, and Daniel J.V. Gonçalves 2014. Relating gaming habits with student performance in a gamified learning experience. In Proceedings of the first ACM SIGCHI annual symposium on Computer-human interaction in play - CHI PLAY '14. ACM, 17-25. DOI: http://dx.doi.org/10.1145/2658537.2658692
[8] Richard Bartle. 1996. Hearts, Clubs, Diamonds, Spades: Players who suit MUDs. fournal of MUD Research 1, 1 (1996).

[9] Djallel Bouneffouf. 2013. DRARS, A Dynamic Risk-Aware Recommender System. $\mathrm{Ph} . D$. Dissertation. Institut National des Télécommunications.

[10] Peter Brusilovski, Alfred Kobsa, and Wolfgang Nejdl (Eds.). 2007. The Adaptive Web: Methods and Strategies of Web Personalization. Springer, Berlin.

[11] Robin Burke. 2007. Hybrid Web Recommender Systems. In The Adaptive Web: Methods and Strategies of Web Personalization, Peter Brusilovski, Alfred Kobsa, and Wolfgang Nejdl (Eds.). Springer, Chapter 12, 377-408.

[12] Marc Busch, Elke Mattheiss, Rita Orji, Andrzej Marczewski, Wolfgang Hochleitner, Michael Lankes, Lennart E. Nacke, and Manfred Tscheligi. 2015. Personalization in Serious and Persuasive Games and Gamified Interactions. In Proceedings of the 2015 Annual Symposium on Computer-Human Interaction in Play - CHI PLAY '15. ACM, 811-816. DOI : http://dx.doi.org/10.1145/2793107.2810260

[13] Robert B Cialdini. 2001. Harnessing the Science of Persuasion. Harvard Business Review 79, 9 (2001), 72-81.

[14] Thomas M Connolly, Elizabeth A Boyle, Ewan MacArthur, Thomas Hainey, and James M Boyle. 2012. A systematic literature review of empirical evidence on computer games and serious games. Computers \& Education 59, 2 (2012), 661-686. DOI : http://dx.doi.org/10.1016/j.compedu.2012.03.004

[15] Paul T Costa and Robert R McCrae. 1998. Trait theories of personality. In Advanced Personality, D F Barone, M Hersen, and V B Van Hasselt (Eds.). Springer, $103-121$.

[16] Edward L Deci and Richard M Ryan. 1985. Intrinsic Motivation and SelfDetermination in Human Behavior. Plenum, New York and London.

[17] Christian Desrosiers and George Karypis. 2011. A Comprehensive Survey of Neighborhood-based Recommendation Methods. In Recommender Systems Handbook, Bracha Shapira, Francesco Ricci, Paul B Kantor, and Lior Rokach (Eds.). Springer, Chapter 4, 107-144.

[18] Sebastian Deterding. 2015. The Lens of Intrinsic Skill Atoms: A Method for Gameful Design. Human-Computer Interaction 30, 3-4 (2015), 294-335. DOI: http://dx.doi.org/10.1080/07370024.2014.993471

[19] Sebastian Deterding, Dan Dixon, Rilla Khaled, and Lennart E Nacke. 2011. From Game Design Elements to Gamefulness: Defining "Gamification". In Proceedings of the $15^{\text {th }}$ International Academic MindTrek Conference. ACM, ACM, Tampere, Finland, 9-15. DOI : http://dx.doi.org/10.1145/2181037.2181040

[20] Lauren S Ferro, Steffen P Walz, and Stefan Greuter. 2013. Towards personalised, gamified systems: an investigation into game design, personality and player typologies. In Proceedings of The $9^{\text {th }}$ Australasian Conference on Interactive Entertainment: Matters of Life and Death. ACM, 7-12. DOI: http://dx.doi.org/10.1145/2513002.2513024

[21] B.J. Fogg. 2002. Persuasive Technology: Using Computers to Change What We Think and Do. Morgan Kaufmann.

[22] AR Gadiyar. 2014. Gamification 3.0: The Power of Personalization. White paper. Cognizantfis Global Technology (2014).

[23] Susan Gauch, Mirco Speretta, Aravind Chandramouli, and Alessandro Micarelli. 2007. User Profiles for Personalized Information Access. In The Adaptive Web: Methods and Strategies of Web Personalization, Peter Brusilovski, Alfred Kobsa, and Wolfgang Nejdl (Eds.). Springer, Chapter 3, 54-89.

[24] David Geiger and Martin Schader. 2014. Personalized task recommendation in crowdsourcing information systems - Current state of the art. Decision Support Systems 65 (2014), 3-16. DOI: http://dx.doi.org/10.1016/j.dss.2014.05.007

[25] Lewis R Goldberg. 1993. The structure of phenotypic personality traits. American Psychologist 48, 1 (1993), 26. DOI : http://dx.doi.org/10.1037/0003-066X.48.1.26

[26] Sajanee Halko and Julie A Kientz. 2010. Personality and persuasive technology: An exploratory study on health-promoting mobile applications. In International Conference on Persuasive Technology. Springer, 150-161. DOI : http://dx.doi.org/ 10.1007/978-3-642-13226-1_16

[27] Juho Hamari, Jonna Koivisto, and Harri Sarsa. 2014. Does gamification work? - A literature review of empirical studies on gamification. In Proceedings of the Annual Hawaii International Conference on System Sciences. 3025-3034. DOI: http://dx.doi.org/10.1109/HICSS.2014.377

[28] Juho Hamari and Janne Tuunanen. 2014. Player types: A meta-synthesis. Transactions of the Digital Games Research 1, 2 (2014). http://todigra.org/index.php/ todigra/article/view/13

[29] Yuan Jia, Bin Xu, Yamini Karanam, and Stephen Voida. 2016. Personality-targeted Gamification: A Survey Study on Personality Traits and Motivational Affordances. In Proceedings of the $34^{\text {th }}$ Annual ACM Conference on Human Factors in Computing Systems - CHI '16. DOI : http://dx.doi.org/10.1145/2858036.2858515

[30] Daniel Johnson, Sebastian Deterding, Kerri-Ann Kuhn, Aleksandra Staneva, Stoyan Stoyanov, and Leanne Hides. 2016. Gamification for health and wellbeing: A systematic review of the literature. Internet Interventions 6 (nov 2016), 89-106. DOI : http://dx.doi.org/10.1016/j.invent.2016.10.002

[31] Maurits Kaptein, Boris De Ruyter, Panos Markopoulos, and Emile Aarts. 2012. Adaptive persuasive systems: A study of tailored persuasive text messages to reduce snacking. ACM Transactions on Interactive Intelligent Systems (TiiS) 2, 2 (2012), 10. DOI : http://dx.doi.org/10.1145/2209310.2209313 
[32] Maurits Kaptein, Panos Markopoulos, Boris de Ruyter, and Emile Aarts. 2009 Can you be persuaded? Individual differences in susceptibility to persuasion. In IFIP Conference on Human-Computer Interaction. Springer, 115-118. DOI http://dx.doi.org/10.1007/978-3-642-03655-2_13

[33] Maurits Kaptein, Panos Markopoulos, Boris de Ruyter, and Emile Aarts. 2015 Personalizing persuasive technologies: Explicit and implicit personalization using persuasion profiles. International fournal of Human-Computer Studies 77 (2015), 38-51. DOI : http://dx.doi.org/10.1016/j.ijhcs.2015.01.004

[34] Rilla Khaled, Pippin Barr, James Noble, Ronald Fischer, and Robert Biddle. 2006 Our place or mine? Exploration into collectivism-focused persuasive technology design. In International Conference on Persuasive Technology. Springer, 72-83. DOI : http://dx.doi.org/10.1007/11755494_11

[35] Yehuda Koren and Robert Bell. 2011. Advances in Collaborative Filtering. In Recommender Systems Handbook, Bracha Shapira, Francesco Ricci, Paul B Kantor, and Lior Rokach (Eds.). Springer, Chapter 5, 145-186.

[36] Pasquale Lops, Marco De Gemmis, and Giovanni Semeraro. 2011. Content-based Recommender Systems: State of the Art and Trends. In Recommender Systems Handbook, Bracha Shapira, Francesco Ricci, Paul B Kantor, and Lior Rokach (Eds.). Springer, Chapter 3, 73-105.

[37] Andrzej Marczewski. 2015. User Types. In Even Ninja Monkeys Like to Play: Gamification, Game Thinking \& Motivational Design. CreateSpace Independent Publishing Platform, 69-84.

[38] Elke Mattheiss, David Sellitsch, Marc Busch, Wolfgang Hochleitner, Josef Froschauer, and Manfred Tscheligi. 2016. Missing the Forest for the Trees: Balancing Personalization Costs and Benefits in Persuasive Games. In Proceed ings of the International Workshop on Personalization in Persuasive Technology (PPT'16). Salzburg, Austria, 40-43.

[39] Michael Meder and Brijnesh-Johannes Jain. 2014. The Gamification Design Problem. arXiv preprint arXiv:1407.0843 (2014).

[40] Alberto Mora, Daniel Riera, Carina Gonzalez, and Joan Arnedo-Moreno. 2015. A literature review of gamification design frameworks. In $7^{\text {th }}$ International Con ference on Games and Virtual Worlds for Serious Applications (VS-Games). DOI : http://dx.doi.org/10.1109/VS-GAMES.2015.7295760

[41] Benedikt Morschheuser, Karl Werder, Juho Hamari, and Julian Abe. 2017. How to gamify? A method for designing gamification. In Proceedings of the $50^{\text {th }}$ Annual Hawaii International Conference on System Sciences (HICSS). IEEE, Hawaii, USA. http://hdl.handle.net/10125/41308

[42] Lennart E Nacke, Chris Bateman, and Regan L Mandryk. 2014. BrainHex: A Neurobiological Gamer Typology Survey. Entertainment Computing 5, 1 (2014), 55-62. DOI : http://dx.doi.org/10.1016/j.entcom.2013.06.002

[43] Lennart E. Nacke and Sebastian Deterding. 2017. The maturing of gamification research. Computers in Human Behavior (2017). DOI : http://dx.doi.org/10.1016/j. chb.2016.11.062

[44] Oded Nov and Ofer Arazy. 2013. Personality-targeted design: Theory, experimental procedure, and preliminary results. In Proceedings of the 2013 Con ference on Computer Supported Cooperative Work. ACM, 977-984. DOI:http //dx.doi.org/10.1145/2441776.2441887

[45] Rita Orji. 2016. Persuasion and Culture: Individualism-Collectivism and Susceptibility to Influence Strategies. In Proceedings of the International Workshop on Personalization in Persuasive Technology (PPT'16). Salzburg, Austria, 30-39.

[46] Rita Orji, Marc Busch, Arie Dijkstra, Michaela Reisinger, Agnis Stibe, and Man fred Tscheligi. 2016. Personalization in Persuasive Technology. In Adjunct Proceedings of the $11^{\text {th }}$ International Conference on Persuasive Technology. 96-99.

[47] Rita Orji and Regan L Mandryk. 2014. Developing culturally relevant design guidelines for encouraging healthy eating behavior. International fournal of Human-Computer Studies 72, 2 (2014), 207-223. DOI : http://dx.doi.org/10.1016/j. ijhcs.2013.08.012

[48] Rita Orji, Regan L Mandryk, and Julita Vassileva. 2014. Gender and persuasive technology: Examining the persuasiveness of persuasive strategies by gender groups. In The $9^{\text {th }}$ International Conference on Persuasive Technology.

[49] Rita Orji, Regan L Mandryk, and Julita Vassileva. 2015. Gender, Age, and Responsiveness to Cialdini's Persuasion Strategies. In International Conference on
Persuasive Technology. Springer, 147-159.

[50] Rita Orji, Regan L. Mandryk, Julita Vassileva, and Kathrin M. Gerling. 2013. Tailoring persuasive health games to gamer type. In Proceedings of the SIGCHI Conference on Human Factors in Computing Systems - CHI '13. 2467-2476. DOI : http://dx.doi.org/10.1145/2470654.2481341

[51] Rita Orji and Karyn Moffatt. 2016. Persuasive technology for health and wellness: State-of-the-art and emerging trends. Health Informatics fournal (2016). DOI: http://dx.doi.org/10.1177/1460458216650979

[52] Rita Orji, Lennart E. Nacke, and Chrysanne DiMarco. 2017. Towards Personalitydriven Persuasive Health Games and Gamified Systems. In Proceedings of the SIGCHI Conference on Human Factors in Computing Systems. ACM. DOI : http: //dx.doi.org/10.1145/3025453.3025577

[53] Rita Orji, Julita Vassileva, and Regan L. Mandryk. 2014. Modeling the efficacy of persuasive strategies for different gamer types in serious games for health. User Modeling and User-Adapted Interaction 24, 5 (2014), 453-498. DOI : http: //dx.doi.org/10.1007/s11257-014-9149-8

[54] Rita O Orji, Julita Vassileva, and Regan L Mandryk. 2013. Modeling gender differences in healthy eating determinants for persuasive intervention design. In International Conference on Persuasive Technology. Springer, 161-173.

[55] Rajat Paharia. 2013. Loyalty 3.0: How to Revolutionize Customer and Employee Engagement with Big Data and Gamification. McGraw Hill Professional.

[56] Michael J Pazzani and Daniel Billsus. 2007. Content-Based Recommendation Systems. In The Adaptive Web: Methods and Strategies of Web Personalization, Peter Brusilovski, Alfred Kobsa, and Wolfgang Nejdl (Eds.). Springer, Chapter 10, 325-341.

[57] Luca Piras, Guiseppe Valetto, Annapaola Marconi, and Marco Pistore. 2015. Virtual Coaches for Mission-Based Gamified Smart Communities. In Workshop on Personalization in Serious and Persuasive Games and Gamified Interactions. London, UK.

[58] Marigo Raftopoulos, Steffen Walz, and Stefan Greuter. 2015. How enterprises play : Towards a taxonomy for enterprise gamification. In DiGRA 2015.

[59] Francesco Ricci, Lior Rokach, and Bracha Shapira. 2011. Introduction to Recommender Systems Handbook. In Recommender Systems Handbook, Bracha Shapira, Francesco Ricci, Paul B Kantor, and Lior Rokach (Eds.). Springer, 1-29.

[60] Richard M. Ryan and Edward L. Deci. 2000. Self-determination theory and the facilitation of intrinsic motivation, social development, and well-being. The American Psychologist 55, 1 (2000), 68-78. DOI : http://dx.doi.org/10.1037/0003-066X. 55.1 .68

[61] Richard M. Ryan, C. Scott Rigby, and Andrew Przybylski. 2006. The motivational pull of video games: A self-determination theory approach. Motivation and Emotion 30, 4 (2006), 347-363. DOI : http://dx.doi.org/10.1007/s11031-006-9051-8

[62] J Ben Schafer, Dan Frankowski, Jon Herlocker, and Shilad Sen. 2007. Collaborative Filtering Recommender Systems. In The Adaptive Web: Methods and Strategies of Web Personalization, Peter Brusilovski, Alfred Kobsa, and Wolfgang Nejdl (Eds.). Springer, Chapter 9, 291-324.

[63] Katie Seaborn and Deborah I. Fels. 2014. Gamification in theory and action: A survey. International fournal of Human-Computer Studies 74 (2014), 14-31. DOI: http://dx.doi.org/10.1016/j.ijhcs.2014.09.006

[64] Gustavo F. Tondello, Rina R. Wehbe, Lisa Diamond, Marc Busch, Andrzej Marczewski, and Lennart E. Nacke. 2016. The Gamification User Types Hexad Scale. In Proceedings of the 2016 Annual Symposium on Computer-Human Interaction in Play - CHI PLAY '16. ACM, Austin, TX, USA. DOI : http://dx.doi.org/10.1145/ 2967934.2968082

[65] Gustavo F. Tondello, Rina R. Wehbe, and Lennart E. Nacke. 2015. Towards a Personalized Playful Digital Wellness Assistant. In Workshop on Personalization in Serious and Persuasive Games and Gamified Interactions. London, UK.

[66] Rob von Roy and Bieke Zaman. 2015. The inclusion or exclusion of teaching staff in a gamified system: an example of the need to personalize. In Workshop on Personalization in Serious and Persuasive Games and Gamified Interactions. London, UK.

[67] Nick Yee. 2006. Motivations for Play in Online Games. CyberPsychology \& Behavior 9, 6 (2006), 772-775. DOI : http://dx.doi.org/10.1089/cpb.2006.9.772 